IV.

\title{
Abdominal Hysterectomy for Inversion of the Uterus, Fatty Degeneration of the Inverted Portion*.
}

\author{
By A. H. Freeland Barbour, M.D., \\ Lecturer in Gynacology, University of Edinburgh.
}

WhILE changes in the mucous membrane of the inverted uterus have been fully described, I can find no references to changes in the muscular wall. The following case is therefore worthy of record :-

Mrs. R., aged 37, a iii.-para, was admitted to Ward 35, on April 11th, 1905.

Two months before admission, the patient was delivered of a fulltime child. The labour was difficult, and instruments were used, as they had been in the two previous labours. All three children were born dead. In her last labour the patient lost a considerable quantity of blood. She had no pain during the puerperium, but, for the first three days, she was unable to pass urine, and it was not drawn off until the third day. When the doctor was doing this he informed her that the "womb was down." She herself did not notice anything wrong. Six weeks after her confinement she was informed that the "womb was inside out." During the time previous to admission she felt nothing specially wrong. There had been no hæmorrhage.

On admission, vaginal examination showed the pelvis to be justo-minor. In the vagina was a mass, equal to the size of a golf ball, rounded and smooth except at a few points where there was irregularity on the surface. No cervix could be felt. On bimanual examination under chloroform no fundus could be felt above the pubes, and the sound could not be passed up alongside the mass. An attempt was made to replace the fundus by pressure with the fingers in the vagina. This was partially successful, and then the cervix could be felt. It was fixed with volsellæ and pressure was again applied to the fundus, but no further reduction could be effected, and in two days the inverted uterus was projecting as before.

- Communicated to the Edinburgh Obstetrical Society, May 9, 1906. 


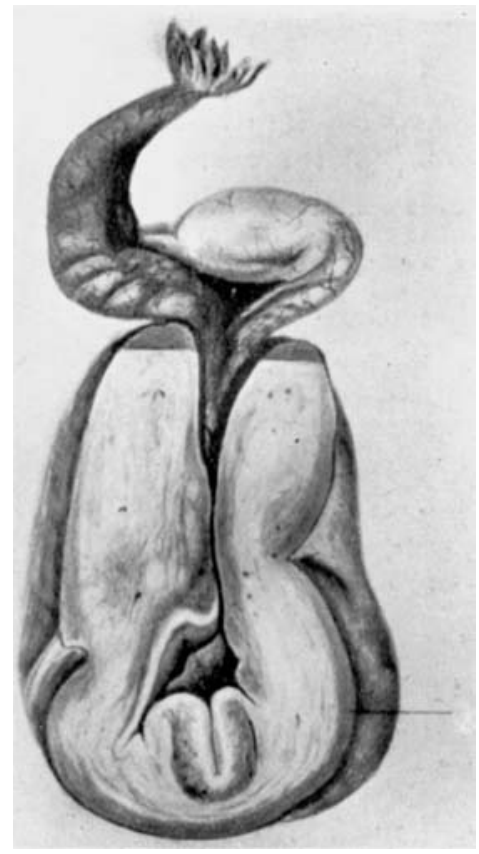

Inverted Iterus split longitudinally. $a=$ Exposed mucous surface. 'The extent of the fatty change in the wall is indicated at the fundus. 
A week later the patient was again anæsthetized and the abdomen was opened. After she had been placed in the Trendelenburg position a cup-shaped depression was visible in the floor of the pelvis across which was a transverse slit with the tubes drawn into it. The posterior rim of the cup was incised through both thicknesses of the inverted wall. Pressure was now again exerted on the inverted fundus from the vagina, and the incision was continued downwards into the cup until the inversion was completely undone by pressure from below. There was very little hæmorrhage from the cut walls of the uterus. The uterus was now seen to be the seat of marked fatty degeneration, and it was determined to remove it. This was done by supravaginal-hysterectomy. One ovary was left.

After the operation the patient was extremely collapsed, and for the first twenty-four hours she suffered from severe shock, which was only controlled by frequent saline enemata. Her convalescence was slow, and was further retarded by an attack of shingles in the right thigh a week after operation. She was discharged well three weeks later.

The specimen (see drawing) consists of the uterus amputated above the cervix, together with the right tube and ovary. The posterior wall of the uterus is split from a point just below the fundus down to the level of amputation. This incision gapes when the uterus is in its normal state, but when the uterus is inverted (which it is readily), the edges of the incision remain in close apposition. When the uterus is thus inverted the ovary and fimbriated end of the tube remain outside the peritoneal cup.

The uterus measures $5.5 \mathrm{~cm}$. in length. The circumference at the line of amputation, which corresponded to the constricting ring, is $5 \mathrm{~cm}$., while the circumference at the widest part of the fundus touching both the tube corners is $12 \mathrm{~cm}$. This accounts for the impossibility of manual reduction.

The exposed mucous surface is smooth and hard, is pale in colour and shows no hæmorrhages. The tube openings are visible and patent.

The peritoneal surface is thrown into longitudinal folds, and there is a deposit of fat visible under it, while the superficial layers of the muscular wall also have undergone fatty change.

Microscopic Examination of Uterine Wall. The exposed surface of the uterus is devoid of mucous membrane. On the surface is a condensed layer of fibrous tissue without any epithelial covering. 
At places there is some débris of broken-down cells. There is no indication of the formation of a stratified squamous epithelium, such as has been described in some cases. No glands are present.

Under this free surface the muscle fibres are degenerated, the nuclear staining being faint. Between the fibres there are many small blood extravasations, some recent, others older and only represented by blood pigment. Beyond this area the muscle fibres are healthy to the depth of the line of large vessels in the uterine wall. On the peritoneal side of these, however, the fibres are extremely degenerated; nuclear staining is absent, and the protoplasm has undergone fatty change. This becomes more marked towards the peritoneal surface until immediately under it the muscle is entirely replaced by fat. The peritoneal coat is thickened in places by a deposit of lymph.

It is difficult to explain why the degeneration has especially affected the outer third of the muscular wall. Greater compression might cause such a change in the wall in the neighbourhood of the inversion ring; but this cause does not operate at the fundus in the bottom of the inverted cup.

This fatty degeneration of the uterine wall has an important bearing on the treatment of inversion.

Küstner was the first to advocate and carry out the operation of hysterectomy in the treatment of cases of inversion in which manual reposition failed. He operated by the vaginal route, gaining access to the peritoneal cavity by a transverse incision through the posterior fornix. The finger was then passed into the inversion cup, and the posterior uterine wall was divided longitudinally until the inversion could be undone. Piccoli also devised an operation along the same lines.

Dührssen operated also by the vaginal route, but incised the anterior fornix and split the anterior wall of the uterus. Other operators have followed him.

In 1901 Dr. Haultain described at the Edin. Obst. Society an operation which he had performed for a case of chronic inversion, in which he opened the abdomen and incised the posterior wall of the uterus. Dr. Haultain's method was follow'ed in the case just recorded. He points out in his paper that the abdominal route has three advantages over the vaginal-(1) the incision in the uterus is reduced to a minimum; (2) traction on the round and broad ligaments may help 
reposition; (3) the uterine wall can be more accurately sutured and hæmorrhage more efficiently controlled.

The present case adds a further argument for the abdominal route, for the fatty change here present, though it must be of rare occurrence, as I can find no references to it in the literature, would more easily escape notice in the vaginal operation.

The abdominal route allowed one to see plainly the condition of the uterine wall, and to decide on hysterectomy as being the best if not the only thing to be done. It is doubtful whether, with the walls in this condition, healing by first intention would have occurred. And even had it occurred, the degeneration of the uterine wall would have been a cause of ill-health, and might have produced complications in pregnancy. 